\title{
Continuum Mechanics of Space Seen from the Aspect of General Relativity-An Interpretation of the Gravity Mechanism
}

\author{
Yoshinari Minami \\ Advanced Science-Technology Research Organization (Formerly NEC Space Development Division), Yokohama 220-0062, Japan
}

Received: February 15, 2015 / Accepted: March 05, 2015 / Published: March 20, 2015.

\begin{abstract}
A mechanical structure of space is suggested. On the supposition that a space as vacuum has a physical fine structure like continuum, it enables us to apply a continuum mechanics to the so-called "vacuum" of space. A space is an infinite continuum and its structure is determined by Riemannian geometry. Assuming that space is an infinite continuum, the pressure field derived from the geometrical structure of space is newly obtained by applying both continuum mechanics and General Relativity to space. A fundamental concept of space-time is described that focuses on theoretically innate properties of space including strain and curvature. As a trial consideration, gravity can be explained as a pressure field induced by the curvature of space.
\end{abstract}

Key words: General Relativity, continuum mechanics, curvature, strain, space-time, gravity.

\section{Introduction}

Given a priori assumption that space as a vacuum has a physical fine structure like continuum, it enables us to apply a continuum mechanics to the so-called "vacuum" of space. Minami proposed a hypothesis for mechanical property of space-time in 1988 [1]. A primary motive was research in the realm of space propulsion theory. His propulsion principle using the substantial physical structure of space-time is based on this hypothesis [2-10].

In this paper, a fundamental concept of space-time is described that focuses on theoretically innate properties of space including strain and curvature.

Assuming that space as vacuum is an infinite continuum, space can be considered as a kind of transparent elastic field. That is, space as a vacuum performs the motions of deformation such as

Corresponding author: Yoshinari Minami, administrative director, research fields: satellite design and engineering, propulsion theory and propulsion physics, laser propulsion, interstellar navigation theory. E-mail: y-minami@mtj.biglobe.ne.jp. expansion, contraction, elongation, torsion and bending. The latest expanding universe theories (Friedmann, de Sitter, inflationary cosmological model) support this assumption. Space can be regarded as an elastic body like rubber. This conveniently coincides with the precondition of a mechanical structure of space.

General relativity implies that space is curved by the existence of energy (mass energy or electromagnetic energy etc.). General relativity is based on Riemannian geometry. If we admit this space curvature, space is assumed as an elastic body. According to continuum mechanics, the elastic body has the property of the motion of deformation such as expansion, contraction, elongation, torsion and bending. General relativity uses only the curvature of space. Expansion and contraction of space are used in cosmology, and a theory using torsion has also been studied by Hayasaka [11] and twistor theory as proposed by Roger Penrose [12] to the torsion of space. 


\section{Mechanical Concept of Space}

When we make a comparison between the space on the Earth and outer space throughout the universe, although there seems to be no difference, obviously a different phenomenon occurs. Simply put, an object moves radially inward, that is, drops straight down on the Earth, but in the universe, the object floats and does not move.

The difference between the two phenomena can be explained as whether space is curved or not, that is, whether 20 independent components of a Riemann curvature tensor is zero or not. In essence, the existence of spatial curvature (and curved extent) determines whether the object drops straight down or not. Although the spatial curvature at the surface of the Earth is very small value, i.e., $3.42 \times 10^{-23}\left(1 / \mathrm{m}^{2}\right)$, it is of enough value to produce $1 \mathrm{G}\left(9.8 \mathrm{~m} / \mathrm{s}^{2}\right)$ acceleration. Conversely, the spatial curvature in the universe is zero, therefore any acceleration is not produced. Accordingly, if the spatial curvature of a localized area including object is controlled to curvature $3.42 \times 10^{-23}\left(1 / \mathrm{m}^{2}\right)$ with an extent, the object moves and receives $1 \mathrm{G}$ acceleration in the universe. Of course, we are required to control both the magnitude and extent of curvature.

\subsection{Fundamental Concept of Space}

Space is an infinite continuum and its structure is determined by Riemannian geometry. Space satisfies the following conditions:

(1) When the infinitesimal distance regulating the distance between the two points changes by a certain physical action, the change is continuous, and the space maintains a continuum even after its change. Now, the concept of strain of continuum mechanics is very important in order to relate a spatial curvature to a practical force. Because the spatial curvature is a purely geometrical quantity. A strain field is required for the conversion of geometrical quantity to a practical force.
(2) The spatial strain is defined as a localized geometrical structural change of space. It implies a change from flat space involved in zero curvature components to curved Riemann space involved in non-zero curvature components.

(3) Space has the only strain-free natural state, and space always returns to the strain-free natural state, i.e., flat space, when an external physical action causing spatial strain is removed.

(4) Spatial strain means some kinds of structural deformation of space, and a body filling up space is affected by the action from its spatial strain. We must distinguish space from an isolated body. An isolated body occupies an area of space by its movement. Basically, an isolated body can move in space and also can change its position.

(5) In order to keep the continuity of space, the velocity of body filling up space cannot exceed the strain rate of space itself.

Since the subject of our study is a four-dimensional Riemann space as a curved space, we ascribe a great deal of importance to the curvature of space. We a priori accept that the nature of actual physical space is a four-dimensional Riemann space, that is, three dimensional space $\left(x=x^{1}, y=x^{2}, \quad z=x^{3}\right)$ and one dimensional time $\left(\mathrm{w}=\mathrm{ct}=\mathrm{x}^{0}\right)$, where $\mathrm{c}$ is the velocity of light. These four coordinate axes are denoted as $x^{i}(\mathrm{i}=$ $0,1,2,3)$.

The square of the infinitesimal distance " $d s$ " between two infinitely proximate points $x^{i}$ and $x^{i}+d x^{i}$ is given by equation of the form:

$$
d s^{2}=g_{i j} d x^{i} d x^{j}
$$

where $g_{i j}$ is a metric tensor.

The metric tensor $g_{i j}$ determines all the geometrical properties of space and it is a function of this space coordinate. In Riemann space, the metric tensor $g^{i j}$ determines a Riemannian connection coefficient $\Gamma^{i}{ }_{j k}$, and furthermore determines the Riemann curvature tensor $R^{p}{ }_{i j k}$ or $R_{p i j k}$, thus the geometry of space is determined by a metric tensor. 
Riemannian geometry is a geometry which provides a tool to describe curved Riemann space, therefore a Riemann curvature tensor is the principal quantity. All the components of Riemann curvature tensor are zero for flat space and non-zero for curved space. If a non-zero component of Riemann curvature tensor exists, the space is not flat space, but curved space. In curved space, it is well known that the result of the parallel displacement of vector depends on the choice of the path. Further, the components of a vector differ from the initial value, after we displace a vector parallel along a closed curve until it returns to the starting point.

An external physical action such as the existence of mass energy or electromagnetic energy yields the structural deformation of space. In the deformed space region, the infinitesimal distance is given by:

$$
d s^{\prime 2}=g_{i j}^{\prime} d x^{i} d x^{j}
$$

where $g_{i j}^{\prime}$ the metric tensor of deformed space region, and we use the convected coordinates $\left(x^{\prime i}=x^{i}\right)$.

As shown in Fig. 1, if the line element between the arbitrary two near points (A and B) in space region $\mathrm{S}$ (before structural deformation) is defined as $d s=g_{i} d x^{i}$, the infinitesimal distance between the two near points is given by Eq. (1):

$$
d s^{2}=g_{i j} d x^{i} d x^{j}
$$

Let us assume that a space region $S$ is structurally deformed by an external physical action and transformed to space region $T$. In the deformed space region $T$, the line element between the identical two near point ( $\mathrm{A}^{\prime}$ and $\mathrm{B}^{\prime}$ ) of the identical space region newly changes, differs from the length and direction, and becomes $d s^{\prime}=g_{i}^{\prime} d x^{i}$.

Therefore, the infinitesimal distance between the two near points using the convected coordinate $\left(x^{\prime i}=x^{i}\right)$ is given by:

$$
d s^{\prime 2}=g_{i j}^{\prime} d x^{i} d x^{j}
$$

The $g_{i}^{\prime}$ is the transformed base vector from the original vase vector $g^{i}$ and the $g_{i j}^{\prime}$ is the transformed metric tensor from the original metric tensor $g_{i j}$. Since the degree of deformation can be expressed as the change of distance between the two points, we get:

$$
\begin{aligned}
& d s^{\prime 2}-d s^{2}=g_{i j}^{\prime} d x^{i} d x^{j}-g_{i j} d x^{i} d x^{j} \\
& =\left(g_{i j}^{\prime}-g_{i j}\right) d x^{i} d x^{j}=r_{i j} d x^{i} d x^{j}
\end{aligned}
$$

Hence the degree of geometrical and structural deformation can be expressed by the quantity denoted change of metric tensor, i.e.

$$
r_{i j}=g_{i j}^{\prime}-g_{i j}
$$

On the other hand, the state of deformation can be also expressed by the displacement vector "u" (Fig. 1).

From the continuum mechanics [13-16], using the following equations:

$$
\begin{gathered}
d u=g^{i} u_{i: j} d x^{j} \\
d s^{\prime}=d s+d u=d s+g^{i} u_{i: j} d x^{j}
\end{gathered}
$$

We use the usual notation ":" for covariant differentiation. From the usual continuum mechanics, the infinitesimal distance after deformation becomes [13] (see Appendix A):

$$
\begin{aligned}
& d s^{\prime 2}-d s^{2}=r_{i j} d x^{i} d x^{j} \\
& =\left(u_{i: j}+u_{j: i}+u_{: i}^{k} u_{k: j}\right) d x^{i} d x^{j}
\end{aligned}
$$

The terms of higher order than second $u^{k}{ }^{k} u_{k: j}$ can be neglected if the displacement is of small enough value. As the actual physical space can be dealt with the minute displacement from the trial calculation of strain, we get:

$$
r_{i j}=u_{i: j}+u_{j: i}
$$

Whereas, according to the continuum mechanics [13], the strain tensor $e_{i j}$ is given by:

$$
e_{i j}=\frac{1}{2} \cdot r_{i j}=\frac{1}{2} \cdot\left(u_{i: j}+u_{j: i}\right)
$$

So, we get:

$$
d s^{\prime 2}-d s^{2}=\left(g_{i j}^{\prime}-g_{i j}\right) d x^{i} d x^{j}=2 e_{i j} d x^{i} d x^{j}
$$




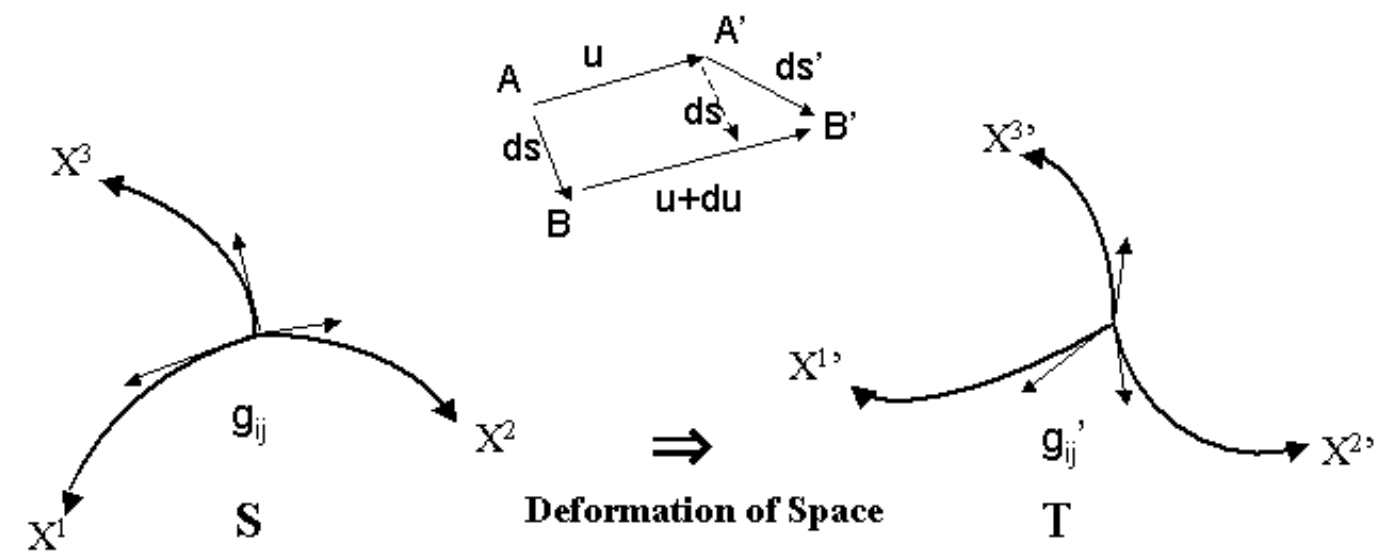

Fig. 1 Fundamental structure of space.

where $g_{i j}^{\prime}, g_{i j}$ is a metric tensor, $e_{i j}$ is a strain tensor, and $d s^{\prime 2}-d s^{2}$ is the square of the infinitesimal distance between two infinitely proximate points $x^{i}$ and $x^{i}+d x^{i}$.

Eq. (11) indicates that a certain geometrical structural deformation of space is shown by the concept of strain. In essence, the change of metric tensor $\left(g_{i j}^{\prime}-g_{i j}\right)$ due to the existence of mass energy or electromagnetic energy tensor produces the strain field $e_{i j}$.

Since space-time is distorted, the infinitesimal distance between two infinitely proximate points $x^{i}$ and $x^{i}+d x^{i}$ is important in our understanding of the geometry of the space-time; the physical strain is generated by the difference of a geometrical metric of space-time. Namely, a certain structural deformation is described by strain tensor $e_{i j}$. From Eq. (11), the strain of space is described as follows:

$$
e_{i j}=1 / 2 \cdot\left(g_{i j}{ }^{\prime}-g_{i j}\right)
$$

It is also worth noting that this result yields the principle of constancy of light velocity in Special Relativity.

\subsection{Mechanics of Space}

Expanding the concept of vector parallel displacement in Riemann space, the following equation has newly been obtained (see Appendix B):

$$
\omega_{\mu v}=R_{\mu v k l} d A^{k l}
$$

where $\omega_{\mu v}$ is rotation tensor, $d A^{k l}$ is infinitesimal areal element.

According to the nature of Riemann curvature tensor $R_{\mu v k l}, \omega_{\mu v}$ indicates the rotation of displacement field. Eq. (13) indicates that a curved space produces the rotation of displacement field in the region of space. Now, the rotation tensor $\omega_{\mu v}$ and strain tensor $e_{i j}$ satisfy the following differential equation in continuum mechanics:

$$
\omega_{\mu v, j}=e_{v j, \mu}-e_{\mu j, v}
$$

This equation is true on condition that the order of differential can be exchanged in a flat space. To expand above equation into a curved Riemann space, the equation shall be transformed to covariant differentiation and it is possible on condition of $\Gamma_{j v}^{\alpha} e_{\mu \alpha}=\Gamma_{j \mu}^{\alpha} e_{v \alpha}$.

Thus, we obtain

$$
\omega_{\mu v: j}=e_{v j: \mu}-e_{\mu j: v}
$$

Here we use the usual notation ":" for covariant differentiation. As is well known, the partial derivative $u_{i, j}=\frac{\partial u_{i}}{\partial x^{j}}$ is not tensor equation. The covariant derivative $u_{i: j}=u_{i, j}-u_{k} \Gamma_{i j}^{k} \quad$ is tensor equation and can be carried over into all coordinate systems. 
Eq. (15) indicates that the displacement gradient of rotation tensor corresponds to difference of the displacement gradient of strain tensor.

Here, if we multiply both sides of Eq. (15) by fourth order tensor denoted the nature of space $E^{i j \mu v}$ formally, we obtain

$$
E^{i j \mu v} \omega_{\mu v: j}=E^{i j \mu v}\left(R_{\mu v k l} d A^{k l}\right)_{: j}=E^{i j \mu v} R_{\mu v k l: j} d A^{k l}
$$

and

$$
\begin{gathered}
E^{i j \mu v} e_{v j: \mu}-E^{i j \mu v} e_{\mu j: v}= \\
\left(E^{i j \mu v} e_{v j}\right)_{: \mu}-\left(E^{i j \mu v} e_{\mu j}\right)_{: v}=\sigma_{: \mu}^{i \mu}-\sigma_{: v}^{i v}=\Delta \sigma_{: r}^{i r}
\end{gathered}
$$

As is well known in the continuum mechanics [13-16], the relationship between stress tensor $\sigma_{i j}$ and strain tensor $e_{m l}$ is given by

$$
\sigma^{i j}=E^{i j m l} e_{m l}
$$

Furthermore, the relationship between body force $F^{i}$ and stress tensor $\sigma_{i j}$ is given by

$$
F^{i}=\sigma^{i j}: j
$$

from the equilibrium conditions of continuum. That is, the elastic force $F^{i}$ is given by the gradient of stress tensor $\sigma_{i j}$.

Therefore, Eq. (17) indicates the difference of body force $\Delta F^{i}$. Accordingly, from Eqs. (16) and (17), the change of body force $\Delta F^{i}\left(=\Delta \sigma^{i r}: r\right)$ becomes

$$
\Delta F^{i}=E^{i j \mu v} R_{\mu v k l: j} d A^{k l}
$$

Here, we assume that $E^{i j \mu v}$ is constant for covariant differentiation, $A^{k l}$ is area element.

The stress tensor $\sigma_{i j}$ is a surface force and $F^{i}$ is a body force. The body force is an equivalent gravitational action because of acting all elements of space uniformly.

Eq. (20) indicates that the gradient of Riemann curvature tensor implying space curvature produces the body force as a space strain force. The non-zero component of Eq. (20) is just only one equation as follows:

$$
\begin{gathered}
F^{3}=F=E^{3330}\left(R_{3030} A^{30}\right)_{: 3} \\
=E^{3330} \cdot \partial\left(R_{3030} A^{30}\right) / \partial r
\end{gathered}
$$

\subsection{Generation of Surface Force Induced by Spatial} Curvature

On the supposition that space is an infinite continuum, continuum mechanics can be applied to the so-called "vacuum" of space. This means that space can be considered as a kind of transparent field with elastic properties. That is, space as a vacuum has the elastic properties of expansion, contraction, elongation, torsion and bending. The latest expanding universe theory (Friedmann, de Sitter, inflationary cosmological model) supports this assumption. We can regard the space of the cosmos as an infinite elastic body like rubber.

If space curves, then an inward normal stress " $-\mathrm{P}$ " is generated. This normal stress, i.e. surface force serves as a sort of pressure field.

$$
-P=N \cdot\left(2 R^{00}\right)^{1 / 2}=N \cdot\left(1 / R_{1}+1 / R_{2}\right)
$$

where $N$ is the line stress, $R_{1}, R_{2}$ are the radius of principal curvature of curved surface, and $R^{00}$ is the major component of spatial curvature.

A large number of curved thin layers form the unidirectional surface force, i.e. acceleration field. Accordingly, the spatial curvature $R^{00}$ produces the acceleration field $\alpha$.

The fundamental three-dimensional space structure is determined by quadratic surface structure. Therefore, a Gaussian curvature $\mathrm{K}$ in two-dimensional Riemann space is significant. The relationship between $\mathrm{K}$ and the major component of spatial curvature $R^{00}$ is given by:

$$
K=\frac{R_{1212}}{\left(g_{11} g_{22}-g_{12}{ }^{2}\right)}=\frac{1}{2} \cdot R^{00}
$$

where $R_{1212}$ is non-zero component of Riemann curvature tensor.

It is now understood that the membrane force on 


\section{Continuum Mechanics of Space Seen from the Aspect of General Relativity-An Interpretation of the Gravity Mechanism}

the curved surface and each principal curvature generates the normal stress "-P" with its direction normal to the curved surface as a surface force. The normal stress $-\mathrm{P}$ acts towards the inside of the surface as shown in Fig. 2a.

A thin-layer of curved surface will take into consideration within a spherical space having a radius of $R$ and the principal radii of curvature that are equal to the radius $\left(R_{1}=R_{2}=R\right)$. Since the membrane force $N$ (serving as the line stress) can be assumed to have a constant value, Eq. (22) indicates that the curvature $R^{00}$ generates the inward normal stress $P$ of the curved surface. The inwardly directed normal stress serves as a pressure field.

When the curved surfaces are included in a great number, some type of unidirectional pressure field is formed. A region of curved space is made of a large number of curved surfaces and they form the field as a unidirectional surface force (i.e. normal stress). Since the field of the surface force is the field of a kind of force, the force accelerates matter in the field, i.e. we can regard the field of the surface force as the acceleration field. A large number of curved thin layers form the unidirectional acceleration field (Fig. 2b). Accordingly, the spatial curvature $R^{00}$ produces the acceleration field $\alpha$. Therefore, the curvature of space plays a significant role to generate pressure field.

Applying membrane theory, the following equilibrium conditions are obtained in quadratic surface, given by:

$$
N^{\alpha \beta} b_{\alpha \beta}+P=0
$$

where $N^{\alpha \beta}$ is a membrane force, i.e. line stress of curved space, $b_{\alpha \beta}$ is second fundamental metric of curved surface, and $P$ is the normal stress on curved surface [13].

The second fundamental metric of curved space $b_{\alpha \beta}$ and principal curvature $K_{(i)}$ has the following relationship using the metric tensor $g_{\alpha \beta}$,

$$
b_{\alpha \beta}=K_{(i)} g_{\alpha \beta}
$$

Therefore we get:

$$
\begin{aligned}
N^{\alpha \beta} b_{\alpha \beta} & =N^{\alpha \beta} K_{(i)} g_{\alpha \beta}= \\
g_{\alpha \beta} N^{\alpha \beta} K_{(i)} & =N_{\alpha}{ }^{\alpha} K_{(i)}=N \cdot K_{(i)}
\end{aligned}
$$

From Eq. (24) and Eq. (26), we get:

$$
N_{\alpha}^{\alpha} K_{(i)}=-P
$$

As for the quadratic surface, the indices $\alpha$ and $i$ take two different values, i.e. 1 and 2, therefore Eq. (27) becomes:

$$
N_{1}^{1} K_{(1)}+N_{2}^{2} K_{(2)}=-P
$$

where $K_{(1)}$ and $K_{(2)}$ are principal curvature of curved surface and are inverse number of radius of principal curvature (i.e. $1 / R_{1}$ and $1 / R_{2}$ ).

The Gaussian curvature $K$ is represented as:

$$
K=K_{(1)} \cdot K_{(2)}=\left(1 / R_{1}\right) \cdot\left(1 / R_{2}\right)
$$

Accordingly, suppose $N_{1}{ }^{1}=N_{2}{ }^{2}=N$, we get:

$$
N \cdot\left(1 / R_{1}+1 / R_{2}\right)=-P
$$

It is now understood that the membrane force on the curved surface and each principal curvature generate the normal stress " $-P$ " with its direction normal to the curved surface as a surface force. The normal stress -P is towards the inside of surface as showing in Fig. 2.

A thin-layer of curved surface will be taken into consideration within a spherical space having a radius of $\mathrm{R}$ and the principal radii of curvature which are equal to the radius $\left(R_{1}=R_{2}=R\right)$. From Eqs. (23) and (29), we then get:

$$
K=\frac{1}{R_{1}} \cdot \frac{1}{R_{2}}=\frac{1}{R^{2}}=\frac{R^{00}}{2}
$$

Considering $N \cdot(2 / R)=-P$ of Eq. (30), and substituting Eq. (31) into Eq. (30), the following equation is obtained:

$$
-P=N \cdot \sqrt{2 R^{00}}
$$

Since the membrane force $\mathrm{N}$ (serving as the line stress) can be assumed to have a constant value, Eq. (32) indicates that the curvature $R^{00}$ generates the 

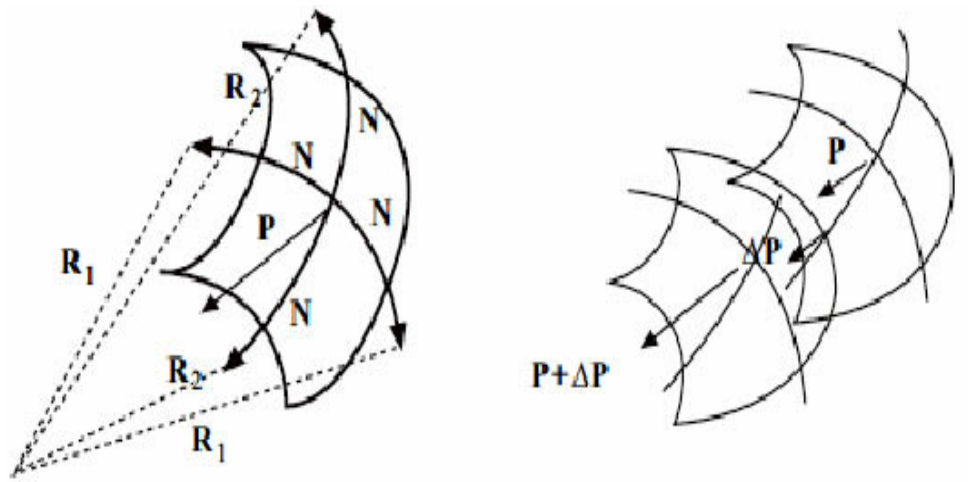

(a)

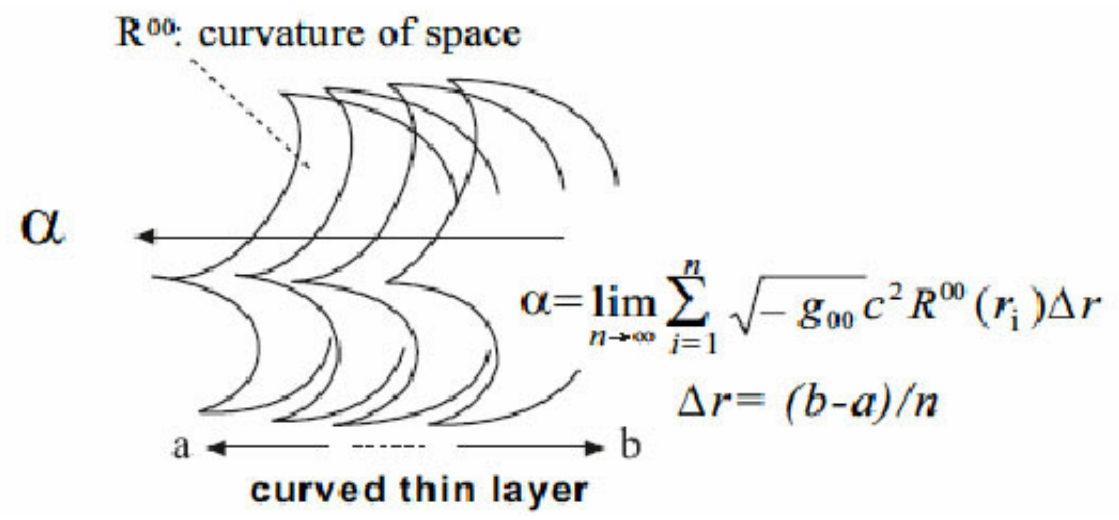

(b)

Fig. 2 Curvature of Space: (a) curvature of space plays a significant role. If space curves, then inward stress (surface force) "P" is generated $\Rightarrow$ A sort of pressure field; (b) a large number of curved thin layers form the unidirectional surface force, i.e. acceleration field $\alpha$.

inward normal stress $\mathrm{P}$ of the curved surface. The inwardly directed normal stress serves as a kind of pressure field. When the curved surfaces are included in great number, some type of unidirectional pressure field is formed. A region of curved space is made of a large number of curved surfaces and they form the field of unidirectional surface force (i.e. normal stress). Since the field of surface force is the field of a kind of force, a body in the field is accelerated by the force, i.e. we can regard the field of surface force as the acceleration field. Accordingly, the cumulated curved region of curvature $R^{00}$ produces the acceleration field $\alpha$.

Here, we give an account of curvature $R^{00}$ in advance. The solution of metric tensor $g^{\mu v}$ is found by gravitational field equation as the following:

$$
R^{\mu v}-\frac{1}{2} \cdot g^{\mu v} R=-\frac{8 \pi G}{c^{4}} \cdot T^{\mu v}
$$

where $R^{\mu v}$ is the Ricci tensor, $R$ is the scalar curvature, $G$ is the gravitational constant, $c$ is the velocity of light, $T^{\mu \nu}$ is the energy momentum tensor. Furthermore, we have the following relation for scalar curvature $R$ :

$$
\begin{gathered}
R=R_{\alpha}^{\alpha}=g^{\alpha \beta} R_{\alpha \beta}, \\
R^{\mu v}=g^{\mu \alpha} g^{\nu \beta} R_{\alpha \beta}, \\
R_{\alpha \beta}=R^{j}{ }_{\alpha j \beta}=g^{i j} R_{i \alpha j \beta}
\end{gathered}
$$

Ricci tensor $R^{\mu \nu}$ is represented by:

$$
R_{\mu \nu}=\Gamma_{\mu \alpha, v}^{\alpha}-\Gamma_{\mu v, \alpha}^{\alpha}-\Gamma_{\mu \nu}^{\alpha} \Gamma_{\alpha \beta}^{\beta}+\Gamma_{\mu \beta}^{\alpha} \Gamma_{v \alpha}^{\beta} \quad\left(=R_{v \mu}\right)
$$

where $\Gamma_{j k}^{i}$ is Riemannian connection coefficient.

If the curvature of space is very small, the term of higher order than the second can be neglected, and Ricci tensor becomes:

$$
R_{\mu v}=\Gamma_{\mu \alpha, v}^{\alpha}-\Gamma_{\mu v, \alpha}^{\alpha}
$$




\section{Continuum Mechanics of Space Seen from the Aspect of General Relativity-An Interpretation of the Gravity Mechanism}

The major curvature of Ricci tensor $(\mu=v=0)$ is calculated as follows:

$$
R^{00}=g^{00} g^{00} R_{00}=-1 \times-1 \times R_{00}=R_{00}
$$

As previously mentioned, Riemannian geometry is a geometry that deals with a curved Riemann space, therefore a Riemann curvature tensor is the principal quantity. All components of Riemann curvature tensor are zero for flat space and non-zero for curved space. If an only non-zero component of Riemann curvature tensor exists, the space is not flat space but curved space. Therefore, the curvature of space plays a significant role.

\subsection{Acceleration Induced by Spatial Curvature}

A massive body causes the curvature of space-time around it, and a free particle responds by moving along a geodesic in that space-time. The path of free particle is a geodesic line in space-time and is given by the following geodesic equation;

$$
\frac{d^{2} x^{i}}{d \tau^{2}}+\Gamma_{j k}^{i} \cdot \frac{d x^{j}}{d \tau} \cdot \frac{d x^{k}}{d \tau}=0
$$

where $\Gamma^{i}{ }_{j k}$ is Riemannian connection coefficient, $\tau$ is proper time, $x^{i}$ is four-dimensional Riemann space, that is, three dimensional space $\left(x=x^{1}, y=x^{2}\right.$, $\left.\mathrm{z}=\mathrm{x}^{3}\right)$ and one dimensional time $\left(\mathrm{w}=\mathrm{ct}=\mathrm{x}^{0}\right), c$ is the velocity of light. These four coordinate axes are denoted as $x^{i}(i=0,1,2,3)$.

Proper time is the time to be measured in a clock resting for a coordinate system. We have the following relation derived from an invariant line element $d s^{2}$ between Special Relativity (flat space) and General Relativity (curved space):

$$
d \tau=\sqrt{-g_{00}} d x^{0}=\sqrt{-g_{00}} c d t
$$

From Eq. (38), the acceleration of free particle is obtained by

$$
\alpha^{i}=\frac{d^{2} x^{i}}{d \tau^{2}}=-\Gamma_{j k}^{i} \cdot \frac{d x^{j}}{d \tau} \cdot \frac{d x^{k}}{d \tau}
$$

As is well known in General Relativity, in the curved space region, the massive body "m (kg)" existing in the acceleration field is subjected to the following force $F^{i}(\mathrm{~N})$ :

$$
\begin{gathered}
F^{i}=m \Gamma_{j k}^{i} \cdot \frac{d x^{j}}{d \tau} \cdot \frac{d x^{k}}{d \tau}= \\
m \sqrt{-g_{00}} c^{2} \Gamma_{j k}^{i} u^{j} u^{k}=m \alpha^{i}
\end{gathered}
$$

where $u^{j}, u^{k}$ are the four velocity, $\Gamma_{j k}^{i}$ is the Riemannian connection coefficient, and $\tau$ is the proper time.

From Eqs. (40) and (41), we obtain:

$$
\begin{aligned}
\alpha^{i} & =\frac{d^{2} x^{i}}{d \tau^{2}}=-\Gamma_{j k}^{i} \cdot \frac{d x^{j}}{d \tau} \cdot \frac{d x^{k}}{d \tau} \\
& =-\sqrt{-g_{00}} c^{2} \Gamma^{i}{ }_{j k} u^{j} u^{k}
\end{aligned}
$$

Eq. (42) yields a more simple equation from the condition of linear approximation, that is, weak-field, quasi-static, and slow motion (speed $\mathrm{v}<<$ speed of light c: $u^{0} \approx 1$ ):

$$
\alpha^{i}=-\sqrt{-g_{00}} \cdot c^{2} \Gamma_{00}^{i}
$$

On the other hand, the major component of spatial curvature $R^{00}$ in the weak field is given by

$$
\begin{gathered}
R^{00} \approx R_{00}=R_{0 \mu 0}^{\mu}= \\
\partial_{0} \Gamma_{0 \mu}^{\mu}-\partial_{\mu} \Gamma_{00}^{\mu}+\Gamma_{0 \mu}^{v} \Gamma_{v 0}^{\mu}-\Gamma_{00}^{v} \Gamma_{v \mu}^{\mu}
\end{gathered}
$$

In the nearly Cartesian coordinate system, the value of $\Gamma_{v \rho}^{\mu}$ are small, so we can neglect the last two terms in Eq. (44), and using the quasi-static condition we get

$$
R^{00} \approx-\partial_{\mu} \Gamma_{00}^{\mu}=-\partial_{i} \Gamma_{00}^{i}
$$

From Eq. (45), we get formally

$$
\Gamma_{00}^{i}=-\int R^{00}\left(x^{i}\right) d x^{i}
$$

Substituting Eq. (46) into Eq. (43), we obtain

$$
\alpha^{i}=\sqrt{-g_{00}} c^{2} \int R^{00}\left(x^{i}\right) d x^{i}
$$

Accordingly, from the following linear approximation scheme for the gravitational field equation: (1) weak gravitational field, i.e. small 
curvature limit, (2) quasi-static, (3) slow-motion approximation (i.e., $v / c<<1$ ), and considering range of curved region, we get the following relation between acceleration of curved space and curvature of space:

$$
\alpha^{i}=\sqrt{-g_{00}} c^{2} \int_{a}^{b} R^{00}\left(x^{i}\right) d x^{i}
$$

where $\alpha^{i}$ : acceleration $\left(\mathrm{m} / \mathrm{s}^{2}\right), g_{00}$ : time component of metric tensor, a-b: range of curved space region (m), $x^{i}$ : components of coordinate $(i=0,1,2,3), c$ : velocity of light, $R^{00}$ : major component of spatial curvature $\left(1 / \mathrm{m}^{2}\right)$.

Eq. (48) indicates that the acceleration field $\alpha^{i}$ is produced in curved space. The intensity of acceleration produced in curved space is proportional to the product of spatial curvature $R^{00}$ and the length of curved region.

Eq. (41) yields more simple equation from above-stated linear approximation $\left(u^{0} \approx 1\right)$,

$$
\begin{gathered}
F^{i}=m \sqrt{-g_{00}} c^{2} \Gamma_{00}^{i}= \\
m \alpha^{i}=m \sqrt{-g_{00}} c^{2} \int_{a}^{b} R^{00}\left(x^{i}\right) d x^{i}
\end{gathered}
$$

Setting $i=3$ (i.e., direction of radius of curvature: $\mathrm{r}$ ), we get Newton's second law:

$$
\begin{gathered}
F^{3}=F=m \alpha= \\
m \sqrt{-g_{00}} c^{2} \int_{a}^{b} R^{00}(r) d r=m \sqrt{-g_{00}} c^{2} \Gamma_{00}^{3}
\end{gathered}
$$

The acceleration $(\alpha)$ of curved space and its Riemannian connection coefficient $\left(\Gamma_{00}^{3}\right)$ are given by:

$$
\alpha=\sqrt{-g_{00}} c^{2} \Gamma_{00}^{3}, \quad \Gamma_{00}^{3}=\frac{-g_{00,3}}{2 g_{33}}
$$

where $c$ : velocity of light, $\mathrm{g}_{00}$ and $\mathrm{g}_{33}$ : component of metric tensor, $g_{00,3}: \partial g_{00} / \partial x^{3}=\partial g_{00} / \partial r$. We choose the spherical coordinates " $\mathrm{ct}=\mathrm{x}^{0}, \mathrm{r}=\mathrm{x}^{3}, \theta=\mathrm{x}^{1}$, $\varphi=x^{2}, \quad$ in space-time. The acceleration $\alpha$ is represented by the equation both in the differential form and in the integral form. Practically, since the metric is usually given by the solution of gravitational field equation, the differential form has been found to be advantageous.

\section{Consideration of Gravity}

Let us consider about gravity. Why does apple fall in the Earth? A well-known answer is that there exists gravity between Earth and apple. Apple is because it's pulled by a law of universal gravitation $F=G \frac{M m}{r^{2}}$ to the Earth. Here, $M$ is the mass of Earth, $\mathrm{m}$ is the mass of apple, $G$ is the gravitational constant, $\mathrm{r}$ is the distance between Earth and apple, $F$ is the gravitational force. From a phenomenological standpoint, it is a sufficient explanation.

However, what is the mechanism? According to General Relativity, it is said that apple moves geodesic line formed by curved space near the Earth. This is seen as lacking in sufficient explanation. The following explanation may allow someone to understand the mechanism of gravity.

If we were to visualize the curvature of space around the Earth (M), we would describe it as having an aggregation of curved surface. A great number of thin curved surfaces are arranged in a spherical concentric pattern. This curvature would gradually become smaller as we moved away from the Earth in what we could imagine as layers of an onion. The surrounding space becomes a flat space of curvature 0 at an imagined immense distance from the Earth (Fig. 3).

In the following thought experiment, an apple of mass $\mathrm{m}$ positioned at a distance $\mathrm{r}$ apart from the Earth would receive a pressure of the field formed by an accumulation of the normal stress (Fig. 3). As was described earlier, with reference to Fig. 2, the membrane force on the curved surface and each principal curvature generates the normal stress " $P$ " with its direction normal to the curved surface as a surface force. The normal stress $-P$ acts towards the inside of the surface as shown in Fig. 2a.

A thin-layer of curved surface will take into consideration within a spherical space having a radius 
of $\mathrm{R}$ and the principal radii of curvature that are equal to the radius $\left(\mathrm{R}_{1}=\mathrm{R}_{2}=\mathrm{R}\right)$. Since the membrane force $N$ (serving as the line stress) can be assumed to have a constant value, the inwardly directed normal stress serves as a pressure field. When the curved surfaces are included in a great number, some type of unidirectional pressure field is formed.

That is, a sort of graduated pressure field is generated by the curved range from an imaginary point "a" in curved space to a point " $b$ " (the point at which space is absent of curvature, i.e., flat space of curvature 0) (Fig. 3). Then apple moves directly towards the center of the Earth, that is, the apple falls. Falling acceleration of apple in curved space is proportional to both the value of spatial curvature and the size of curved space.

If the Earth (M) were to disappear instantly, the curved surface of space close to the Earth would return to the flat surface. Because an external action causing curvature (i.e., mass energy) disappears. The change from a curved surface to a flat surface would advance the position $r$ of the apple at the speed of light (i.e., the strain rate of space-time). The propagation velocity of the change from flat space to curved space and the propagation velocity of change from curved space to flat space are both the same, i.e. the velocity of light.

However, in our thought experiment, the apple would still receive pressure from the surrounding field by the accumulation of the normal stress.

Because, since there still exists the curved region behind the apple from $a$ to $b$ (the remote flat space), the apple continues falling. The pressure continues to push the apple to the center of the Earth (Fig. 4).

However, as soon as the change from a curved surface to a flat surface passes through the point of the apple (i.e., "a" point), the pressure at point "a" disappears and the apple would only float without falling (Fig. 5).

The above discussion provides a basis to consider the following thought experiment. Even if the Sun instantly disappeared, the Earth would still continue to revolve around the Sun until 8 minutes 32 seconds, or the time at which it takes light to advance between the Sun and Earth. However, as soon as the change from curved surface to flat surface passes through the point of the Earth, or at 8 minutes 32 seconds after the event, the pressure pushing the Earth would disappear, and the Earth would fly away in a direction tangential to the revolution of its orbit.

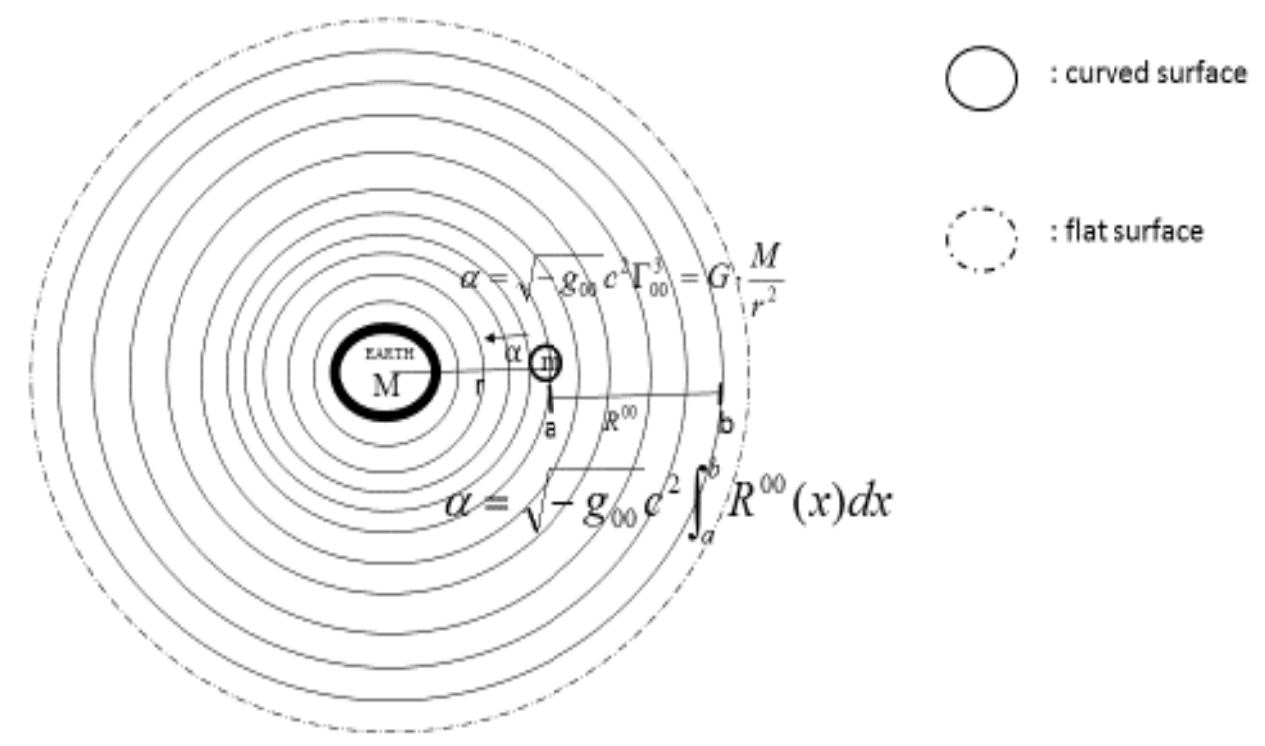

Fig. 3 Apple falls receiving a pressure of the field. 

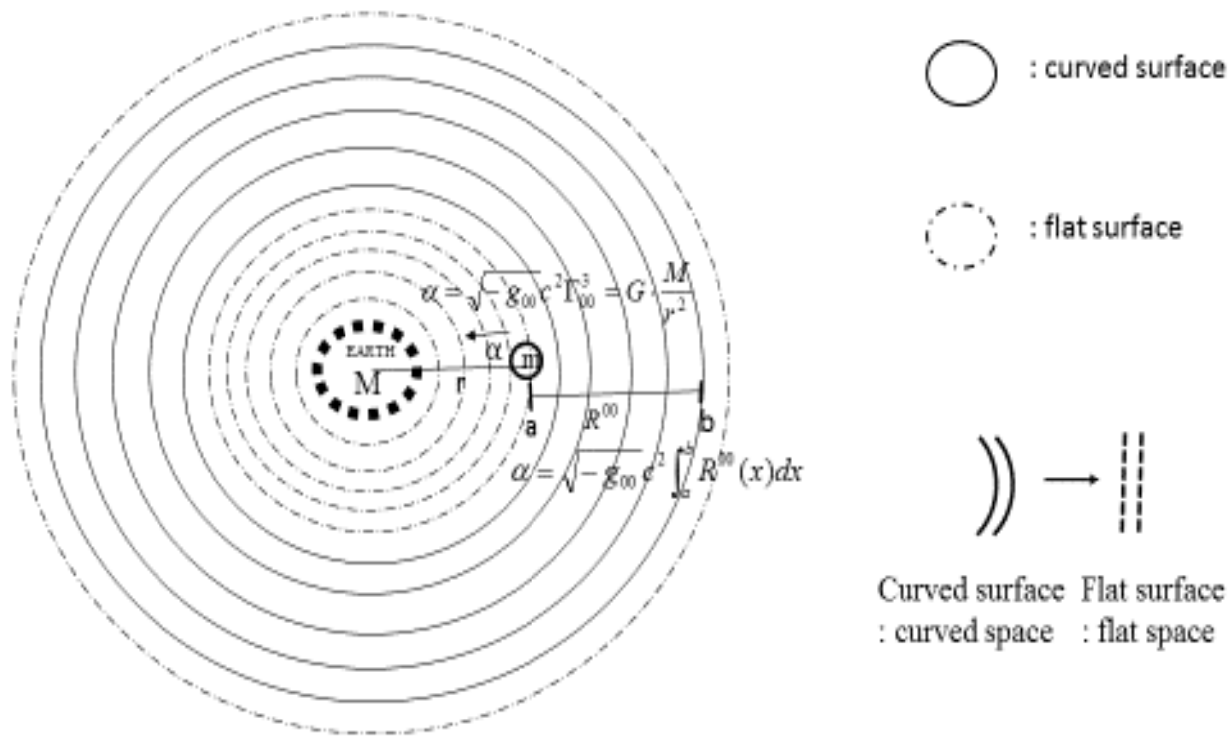

Curved surface Flat surface : curved space : flat space

Fig. 4 Apple still continues falling receiving a pressure of the field.

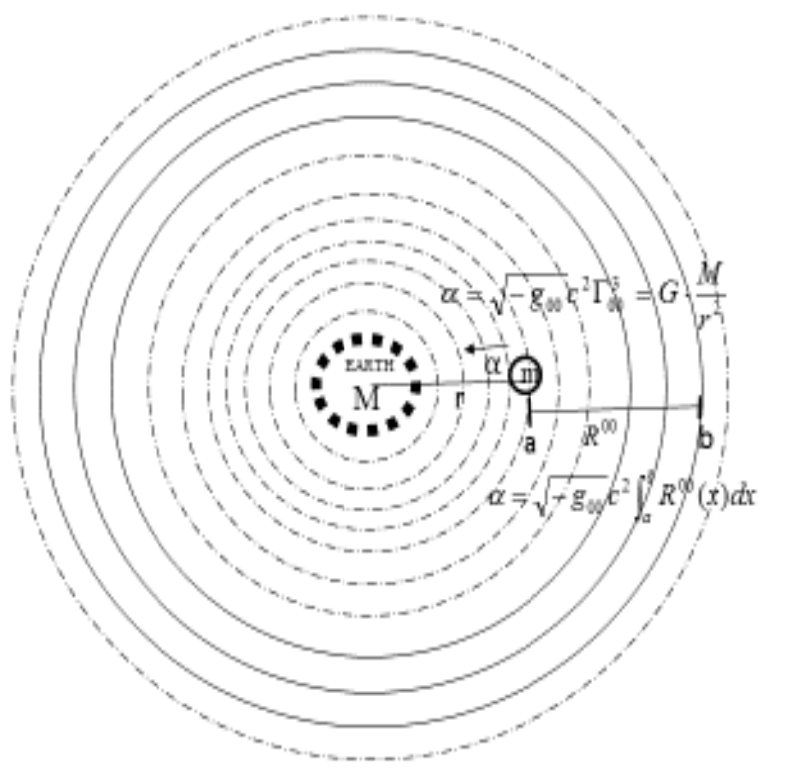

( curved surface

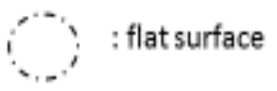<smiles>C#[V]C=C</smiles>

Curved surface Flat surface : curved space : flat space

Fig. 5 Apple only floats without falling due to lack of pressure of the field.

In view of this, gravity may be considered as a pressure generated in a region of curved space.

\section{Conclusion}

Assuming that space is an infinite continuum, a mechanical concept of space became identified. Space can be considered as a kind of transparent elastic field. The pressure field derived from the geometrical structure of space is newly obtained by applying both continuum mechanics and General Relativity to space.
As a result, a fundamental concept of space-time is described that focuses on theoretically innate properties of space including strain and curvature. As a trial consideration, gravity can be explained as a pressure field induced by the curvature of space.

\section{References}

[1] Minami, Y. 1988. "Space Strain Propulsion System." In Proceedings of 16th International Symposium on Space Technology and Science, 125-36.

[2] Minami, Y. 1994. "Possibility of Space Drive Propulsion." 


\section{Continuum Mechanics of Space Seen from the Aspect of General Relativity-An Interpretation of the Gravity Mechanism}

In Proceedings of 45th Congress of the International Astronautical Federation, 658.

[3] Minami, Y. 1997. "Space faring to the Farthest Shores-Theory and Technology of a Space Drive Propulsion System." Journal of the British Interplanetary Society 50: 263-76.

[4] Minami, Y. 2003. "An Introduction to Concepts of Field Propulsion." Journal of the British Interplanetary Society 56: 350-9.

[5] Minami, Y. 2013. "Space Drive Propulsion Principle from the Aspect of Cosmology." Journal of Earth Science and Engineering 3: 379-92.

[6] Minami, Y. 2013. "Basic concepts of space drive propulsion-Another view (Cosmology) of propulsion principle." Journal of Space Exploration 2: 106-15.

[7] Minami, Y., 2014. A Journey to the Stars-By Means of Space Drive Propulsion and Time-Hole Navigation. Berlin: LAMBERT Academic Publishing.

[8] Minami, Y. 1993. "Hyper-Space Navigation Hypothesis for Interstellar Exploration." In Proceedings of 44th Congress of the International Astronautical Federation,

[9] Minami, Y. 2003. "Travelling to the Stars: Possibilities
Given by a Spacetime Featuring Imaginary Time." Journal of the British Interplanetary Society 56: 205-11.

[10] Minami, Y., 2014. "A Space Propulsion Principle Brought about by Locally-Expanded Space.” Journal of Earth Science and Engineering 4: 490-9.

[11] Hayasaka, H. 1994. "Parity Breaking of Gravity and Generation of Antigravity due to the de Rham Cohomology Effect on Object's Spinning." In Proceedings of $3 \mathrm{rd}$ International Conference on Problems of Space, Time, Gravitation,

[12] Huggett, S. A., and Tod, K. P. 1985. An Introduction to Twistor Theory. UK: Cambridge University Press.

[13] Flügge, W. 1972. Tensor Analysis and Continuun Mechanics. New York: Springer-Verlag.

[14] Fung, Y. C. 2001. Classical and Computational Solid Mechanics. New Jersey: World Scientific Publishing Co. Pre. Ltd..

[15] Ziegler, H. 1977. An Introduction to Thermomechanics. Zürich: North-Holland Publishing Company.

[16] Borg, S. F. 1963. Matrix-Tensor Methods in Continuum Mechanics. New York: D. Van Nostrand Company.

\section{Appendix A: Derivation of Eq. (8)}

Let us consider two adjacent spatial points A and B in the unreformed space, Fig. 1, which are the end points of a line element vector $d s$. During the deformation, point A undergoes the displacement $\mathrm{u}$ and moves point A', while point $\mathrm{B}$ experiences a slightly different displacement $u+d u$ when moving to point B'.

From Fig. 1 we read the simple vector equation

$$
d s^{\prime}=d s+d u=d s+g^{i} u_{i: j} d x^{j}=g_{i} d x^{i}+g^{i} u_{i: j} d x^{j}
$$

We may now write the square of the deformed line element. Since all indices are dummies, they have been chosen so that the final result looks best. When we multiply the two factors term by term and switch the notation for some dummy pairs, we obtain:

$$
d s^{\prime} \cdot d s^{\prime}=\left(g_{i} d x^{i}+g^{k} u_{k: i} d x^{i}\right) \cdot\left(g_{j} d x^{j}+g^{l} u_{l: j} d x^{j}\right)=\left(g_{i j}+2 g_{i} \cdot g^{l} u_{l: j}+g^{k l} u_{k: i} u_{l: j}\right) d x^{i} d x^{l}
$$

Using Eq. (3) and Eq. (1), from Eq. (A2), we get:

$$
d s^{\prime 2}-g_{i j} d x^{i} d x^{j}=\left(2 g_{i} \cdot g^{l} u_{l: j}+g^{k l} u_{k: i} u_{l: j}\right) d x^{i} d x^{l}
$$

Left side of Eq. (A3):

$$
g^{\prime}{ }_{i j} d x^{i} d x^{j}-g_{i j} d x^{i} d x^{j}=\left(g^{\prime}{ }_{i j}-g_{i j}\right) d x^{i} d x^{j}=r_{i j} d x^{i} d x^{j}
$$

Right side of Eq. (A3): considering $2 g_{i} g^{l} u_{l: j}=2 \delta_{i}^{l} u_{l: j}=2 u_{i: j}$ and $g^{k l} u_{k: i} u_{l: j}=u_{: i}^{l} u_{l: j}=u_{: i}^{k} u_{k: j}$, (changes of the dummy indices $1 \rightarrow \mathrm{k}$ ), then,

$$
\left(2 g_{i} \cdot g^{l} u_{l: j}+g^{k l} u_{k: i} u_{l: j}\right) d x^{i} d x^{l}=\left(2 u_{i: j}+u_{: i}^{k} u_{k: j}\right)
$$


Since $r_{i j}=r_{j i}$, considering $2 u_{i: j}=u_{i: j}+u_{j: i}$,

$$
\left(g_{i j}^{\prime}-g_{i j}\right) d x^{i} d x^{j}=r_{i j} d x^{i} d x^{j}=\left(u_{i: j}+u_{j: i}+u^{k}: i u_{k: j}\right) d x^{i} d x^{j}
$$

Finally we obtain:

$$
d s^{\prime 2}-d s^{2}=r_{i j} d x^{i} d x^{j}=\left(u_{i: j}+u_{j: i}+u_{: i}^{k} u_{k: j}\right) d x^{i} d x^{j}
$$

\section{Appendix B: Derivation of Eq. (13)}

Let us suppose that covariant vector $A_{i}(P)$ at point $\mathrm{P}(\mathrm{x})$ is transported parallel to $\mathrm{Q}(\mathrm{x}+\mathrm{d} 1 \mathrm{x}+\mathrm{d} 2 \mathrm{x})$ via path $\mathrm{I}$ is $A_{i}(Q)_{\mathrm{I}}$. On the while, covariant vector $A_{i}(P)$ at point $\mathrm{P}(\mathrm{x})$ is transported parallel to $\mathrm{Q}(\mathrm{x}+\mathrm{d} 1 \mathrm{x}+\mathrm{d} 2 \mathrm{x})$ via path II is $A_{i}(Q)_{\amalg} \cdot($ Fig. $\mathrm{B} 1)$.

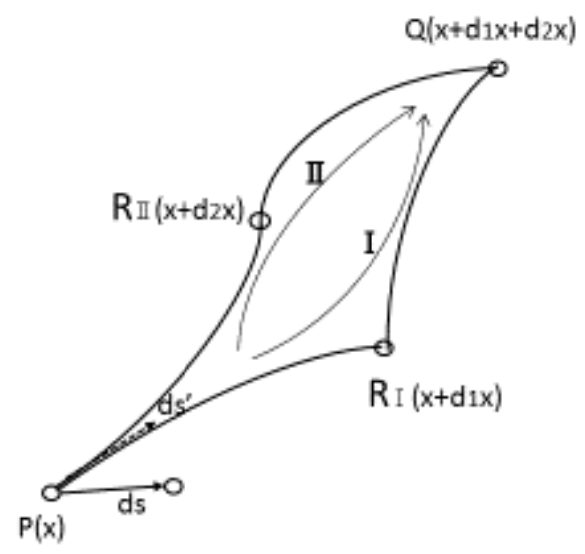

Fig. B1 Parallel transport of vector via two paths.

The result of these parallel transport of vector via two paths does not differ. The difference is indicated by:

$$
A_{i}(Q)_{\mathrm{I}}-A_{i}(Q)_{\amalg}=R^{\rho}{ }_{i k \sigma}(P) A_{\rho}(P) d_{1} x^{k} d_{2} x^{\sigma}
$$

where $R_{i k \sigma}^{\rho}(p)$ is Riemann curvature tensor at point $P$.

Considering $A_{i}(Q)_{\mathrm{I}}+\left(-A_{i}(Q)_{\amalg}\right)$, Eq. (B1) indicates the quantum not returned of vector $A_{i}(P)$ in case that parallel transport of a vector $A_{i}(P)$ along a closed path has been employed at each segment of the loop $\mathrm{P} \rightarrow \mathrm{RI} \rightarrow \mathrm{Q} \rightarrow \mathrm{R} I I \rightarrow \mathrm{P}$ and $\mathrm{ultimately}$ leads back to the point of departure, i.e., original point $\mathrm{P}(\mathrm{x})$ (Fig. B2).

Mathematically, Riemann curvature tensor is the result of a difference that changed the order of the covariant derivatives as seen Eq. (B2), and its non-commutative part is represented by the Riemann curvature tensor.

$$
X_{i: j k}-X_{i: k j}=R_{i j k}^{p} X_{p}
$$

Let us consider point $\mathrm{R}$ adjacent spatial point $\mathrm{P}$, Fig. $\mathrm{B} 2$, which are the end points of a line element vector $d s$. If vector $d s$ at $\mathrm{P}(\mathrm{x})$ is transported parallel to $R I(x+d 1 x)$ and thence to $Q(x+d 1 x+d 2 x)$, then parallel transport from $Q(x+d 1 x+d 2 x)$ to original point $P(x)$ via $\mathrm{R} I I(\mathrm{x}+\mathrm{d} 2 \mathrm{x})$, the result is the new vector $d s^{\prime}$.

Parallel transport of a vector $d s$ along a closed path that ultimately leads back to the point of departure will result in a new vector $d s^{\prime}$ at the original point $\mathrm{P}(\mathrm{x})$; the new vector $d s^{\prime}$ differs from the original vector $d s$, even though the proper procedure for parallel transport has been employed at each segment of the loop. $d s^{\prime}-d s$ indicates the quantum not returned of vector, also is denoted by 
displacement vector $d u$. This arises from nonzero curvature of space.

Another interpretation, two adjacent spatial points $\mathrm{P}$ and $\mathrm{R}$ in the unreformed space, Fig. B2, which are the end points of a line element vector $d s$. During the deformation, $P$ under goes the displacement u and moves $P^{\prime}$, while $R$ experiences a slightly different displacement $u+d u$ when moving to $R^{\prime}$.

These phenomena are equivalent, it is not possible to identify them.

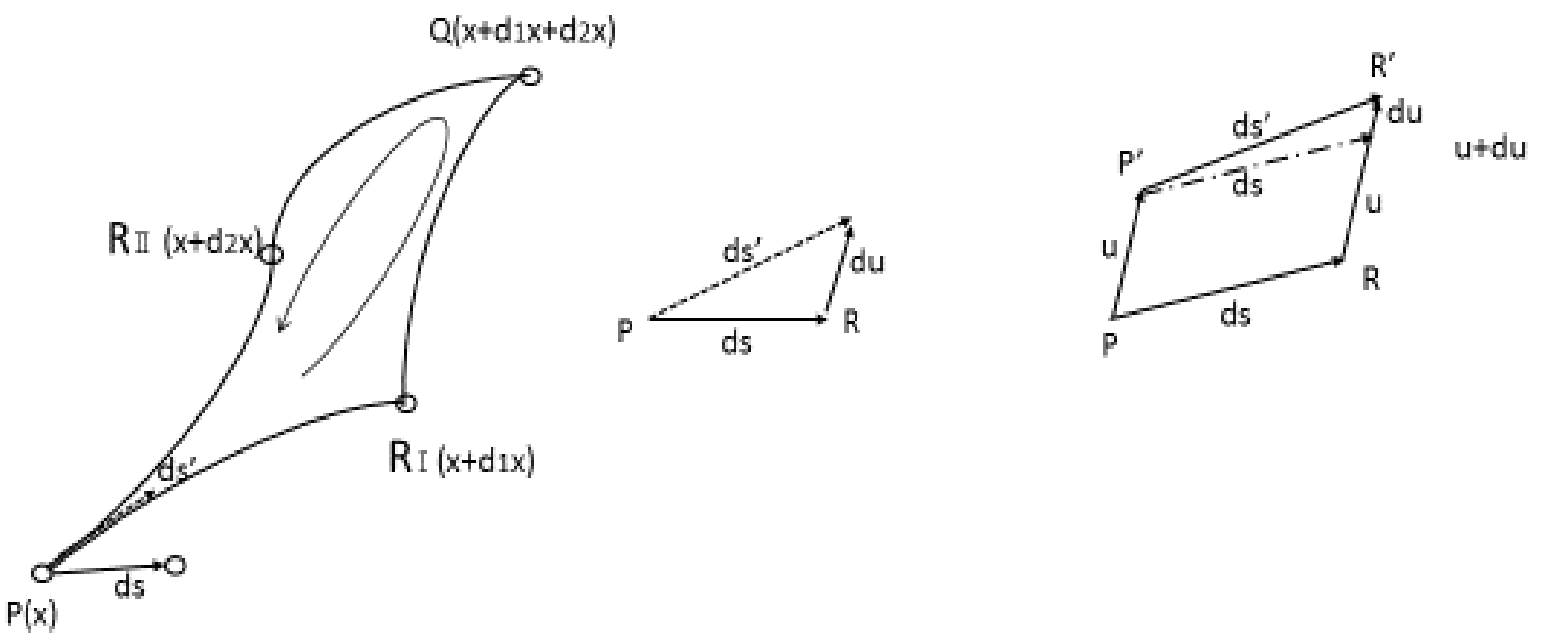

Fig. B2 Parallel transport of vector along a closed path and displacement vector.

From above, we get:

$$
A_{i}(Q)_{\mathrm{I}}-A_{i}(Q)_{\amalg}=d s^{\prime}-d s=R_{i k \sigma}^{\rho}(P) A_{\rho}(P) d_{1} x^{k} d_{2} x^{\sigma}
$$

Since $d s^{\prime}-d s=d u$, infinitesimal displacement vector du is described in

$$
\begin{gathered}
d u=d u_{i}=u_{i: j} d x^{j} \\
A_{i}(Q)_{\mathrm{I}}-A_{i}(Q)_{\amalg}=d s^{\prime}-d s=d u=u_{i: j} d x^{j}
\end{gathered}
$$

Apply a vector $A_{\rho}$ (P) in Eq. (B3) to a line element vector $d s=d s_{\rho}$, from Eq. (B5),

$$
u_{i: j} d x^{j}=R_{i k \sigma}^{\rho} d s_{\rho} d_{1} x^{k} d_{2} x^{\sigma}
$$

Now let us multiply both sides of Eq. (B6) by $g^{r \rho}$, we get following:

$$
\begin{gathered}
g^{r \rho} u_{i: j} d x^{j}=R^{\rho}{ }_{i k \sigma} d s^{r} d_{1} x^{k} d_{2} x^{\sigma}, \text { then, } \\
g^{r \rho} u_{i: j}=R^{\rho}{ }_{i k \sigma} \frac{d s^{r}}{d x^{j}} d_{1} x^{k} d_{2} x^{\sigma}=R^{\rho}{ }_{i k \sigma} \frac{d x^{r}}{d x^{j}} d_{1} x^{k} d_{2} x^{\sigma}=R^{\rho}{ }_{i k \sigma} \delta_{j}^{r} d_{1} x^{k} d_{2} x^{\sigma}, \text { finally } \\
g^{j \rho} u_{i: j}=R^{\rho}{ }_{i k \sigma} d_{1} x^{k} d_{2} x^{\sigma}
\end{gathered}
$$

Multiplying both sides of Eq. (B7) by $g_{\rho m}$,

$$
g_{\rho m} g^{j \rho} u_{i: j}=g_{\rho m} R^{\rho}{ }_{i k \sigma} d_{1} x^{k} d_{2} x^{\sigma} \text {, then applying, } g_{\rho m} g^{j \rho}=\delta_{m}^{j} \text {, we get: }
$$




$$
u_{i: m}=R_{m i k \sigma} d_{1} x^{k} d_{2} x^{\sigma}
$$

For convenience, returns the index $\mathrm{m}$ to $j$,

$$
u_{i: j}=R_{j i k \sigma} d_{1} x^{k} d_{2} x^{\sigma}
$$

Interchanging index $i, j$, we get:

$$
u_{j: i}=R_{i j k \sigma} d_{1} x^{k} d_{2} x^{\sigma}
$$

On the other hand, using the nature of the Riemann curvature tensor,

$$
R_{j i k \sigma}=-R_{i j k \sigma}
$$

Subtracting Eq. (B10) from Eq. (B9), we obtain:

$$
u_{i: j}-u_{j: i}=-R_{i j k \sigma} d_{1} x^{k} d_{2} x^{\sigma}-R_{i j k \sigma} d_{1} x^{k} d_{2} x^{\sigma}=-2 R_{i j k \sigma} d_{1} x^{k} d_{2} x^{\sigma}
$$

By continuum mechanics, anti-symmetric part of the displacement gradient tensor represents the rotation tensor $\omega_{i j}$,

$$
\omega_{i j}=\frac{1}{2}\left(u_{j: i}-u_{i: j}\right)
$$

Accordingly, we obtain:

$$
\omega_{i j}=\frac{1}{2}\left(u_{j: i}-u_{i: j}\right)=R_{i j k \sigma} d_{1} x^{k} d_{2} x^{\sigma}=R_{i j k \sigma} d A^{k \sigma}
$$

where $d A^{k \sigma}$ is the area element enclosed by the vector $d_{1} x^{k}$ and vector $d_{2} x^{\sigma}$.

Thus, changing the dummy of incidences $i, j, k, \sigma \rightarrow \mu, v, k, l$, we get finally:

$$
\omega_{\mu v}=R_{\mu v k l} d A^{k l}
$$

where $\omega_{\mu v}$ is rotation tensor, $d A^{k l}$ is infinitesimal areal element. 